\title{
Kinetic Theory for Particle Concentration Distribution in Two-Phase Flow
}

\author{
By Guangqian Wang' and Jinren $\mathrm{Ni}^{2}$
}

\begin{abstract}
The results of experiments in open channels and closed pipelines show two kinds of patterns for the vertical distribution of particle concentration (i.e., pattern I and pattern $\Pi$ ). The former shows a pattern of maximum concentration at some location above the bottom and the downward decay of the concentration below the location. The latter always shows an increase of the particle concentration downward over the whole vertical, with the maximum value at the bottom. Many investigations were made on the pattern II, but few were made on pattern I. In this paper, a particle velocity distribution function is first obtained in the equilibrium state or in dilute steady state for the particle in two-phase flows, then a theoretical model for the particle concentration distribution is derived from the kinetic theory. More attention is paid to the predictions of the concentration distribution of pattern I and comparisons of the present model are made with the data measured by means of laser doppler anemometry (LDA). Very good agreements are obtained between the measured and calculated results.
\end{abstract}

\section{INTRODUCTION}

There have been numerous investigations into the problems of particle concentration distribution in two-phase flows with two-dimensional steadyflow conditions since Schmidt (Graf 1971) started similar work on dust distribution in the atmosphere during early 1930s. So far, diffusion theory, mixture theory, energy theory, similarity theory, stochastic theory and others have been used to approach the problem of the vertical particle concentration distribution. A review of all these theories and their relationships is given in $\mathrm{Ni}$ and Hui (1988). As a result, a generalized formula on the vertical distribution of the particle concentration was obtained by $\mathrm{Ni}$ (1989), including, for special cases, nearly all the previous formulas derived from different theories.

$\mathrm{Ni}$ and Wang (1987) also found two kinds of patterns of particle concentration distribution, pattern I and pattern II, as shown in Fig. 1, instead of the unique pattern II distribution known to most investigators. A lot of measured data show the existence of pattern I [those reported by Bouvard (1985), Dai (1985), Michalik (1973) and Wang (1989)], but little attention has been paid by the researchers. According to $\mathrm{Ni}$ and Wang (1987), the mechanism for the existence of two kinds of concentration distribution patterns can be explained qualitatively from the angle of fluctuating characteristics of the fluid and particles. To explain the mechanism more precisely, a kinetic theory of two-phase flow for the microscopic descriptions of particles is introduced in this paper and we supply a lot of measured data from the experiments that were specially designed for testing the present model. Noting that numerous work has been done on pattern II, emphasis is laid on the explanations and predictions of pattern $I$ in the following analyses.

${ }^{1}$ Researcher, Inst. of Mech., Chinese Acad. of Sci., Beijing 100080 , China.

${ }^{2}$ Researcher, Dept. of Geography, Peking Univ, Beijing 100871, China.

Note. Discussion open until May 1, 1991. To extend the closing date one month, a written request must be filed with the ASCE Manager of Journals. The manuscript for this paper was submitted for review and possible publication on February 2, 1990. This paper is part of the Journal of Engineering Mechanics, Vol. 116, No. 12, December, 1990. CASCE, ISSN 0733-9399/90/0012-2738/ $\$ 1.00+\$ .15$ per page. Paper No. 25319. 


\section{Velocity Distaibution function for Particles in Dilute Steady TWO-PHASE FLows}

Working much like the ordinary kinetic theory of gases, the kinetic theory of two-phase flow in a liquid-particle system gives us much more information than other theories could. The movement of solid particles can be described by the Boltzmann equation

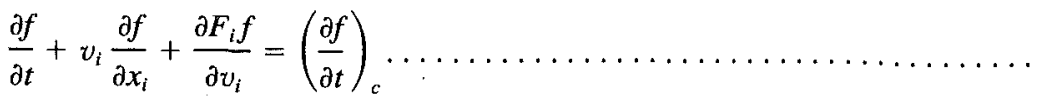

where $f=f\left(v_{i}, x_{i}, t\right)=$ velocity distribution function of particles; $v_{i}=$ stochastic velocity; $x_{i}=$ spatial position; $t=$ time; $F_{i}=$ unit mass force on a particle, including both the gravitational force and the action of the ambient fluid. The right-hand side of Eq. 1 is the collision term, which describes the collisions between solid particles.

The velocity distribution function of particles means the number of particles with velocity between $v_{i}$ and $v_{i}+d v_{i}$ per unit volume is $f d v_{1} d v_{2} d v_{3}$. For two-phase flows, if the velocity distribution function $f$ can be obtained from Boltzmann equation of particles in connection with the equation of motion of the liquid fluid, then exhaustive information, including the issue of concentration distribution, will be considered to be known. However, it is almost impossible to find a solution from Eq. 1 under ordinary flow conditions, owing to the fact that the collision term is a complex integral term of $f$. For flows in equilibrium state or in dilute steady state, the effect of collisions are relatively small, so $(\partial f / \partial t)_{c}$ can be neglected approximately. In such circumstances, Eq. 1 is simplified as

$v_{i} \frac{\partial f}{\partial x_{i}}+\frac{\partial F_{i} f}{\partial v_{i}}=0$

and the velocity distribution function $f$ for particles is thus obtained from Eq. 2 by means of a variational method similar to the treatment for equilibrium state of molecules in the kinetic theory of gases.

In the same spirit of the kinetic theory of gases, the kinetic theory of particle-liquid system can be treated on the basis of the Boltzmann equation of the velocity distribution function. For example, the equilibrium state of molecules in ordinary kinetic theory of gases means a statistical average state of the balanced collisions among the molecules themselves. A similar definition is available for the equilibrium state of particles in two-phase flows,

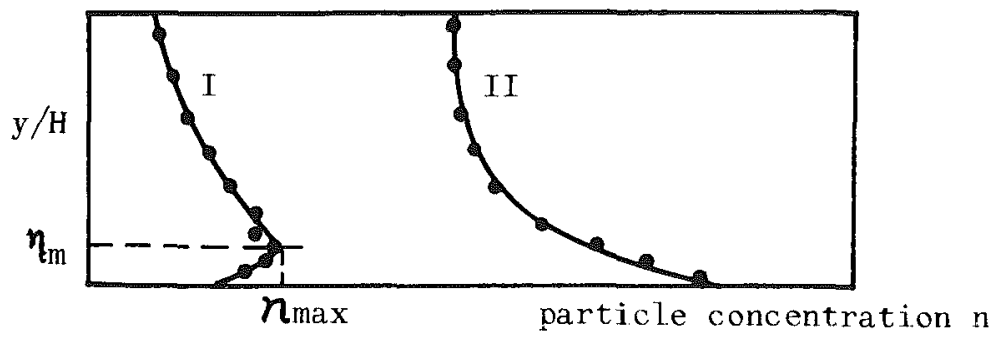

FIG. 1. Two Patterns for Concentration Distribution 
but the equilibrium state in particle-liquid flows means balanced states of collisions among the particles and also interactions between particles and ambient fluid. For the dilute steady state in particle-liquid flows, collisions among the particles seldom occur and an assumption of the equilibrium state in literal meaning can be used, so a function $H(f)$ for particles may be defined in a manner similar to that in kinetic theory of gases (Chapman and Cowling 1970)

$H(f)=\int f\left(v_{i}, x_{i}, t\right) \ln \left[f\left(v_{i}, x_{i}, t\right)\right] d v_{i}$

in which $d v_{i}=d v_{1} d v_{2} d v_{3}$. For equilibrium state, a minimum $H(f)$ will be expected. By utilizing the form of the variation equation, we have

$\delta H(f)=\delta \int f \ln f d v_{i}=0$.

with its additional conditions, such as the conservation of particle-number per unit volume

$\delta n=\delta \int f d v_{i}=0$.

the conservation of momentum

$\delta \int m \mathbf{v} f d v_{i}=0$

and the conservation of kinetic energy

$\delta \int \frac{1}{2} m v^{2} f d v_{i}=0$

Using the Lagrange method with Eq. 5 multiplied by an operator $\lambda^{\prime}$ and making a scalar product of Eq. 6 and the vector $(1 / \mathbf{m}) \mathbf{b}$, and also with Eq. 7 multiplied by $\beta$, the variational problem becomes

$\int\left(1+\ln f-\lambda^{\prime}-\mathbf{b} \cdot \mathbf{v}+\frac{1}{2} m \beta v^{2}\right) d v_{i} \delta f=0$

or

$\ln f=\left(\lambda^{\prime}-1\right)+\mathbf{b} \cdot \mathbf{v}-\frac{1}{2} m \beta v^{2} \quad f=\exp \left(\lambda+\mathbf{b} \cdot \mathbf{v}-\frac{1}{2} m \beta v^{2}\right) \ldots \ldots$

in which $\lambda=\lambda^{\prime}-1$, and both Lagrange operators ( $\beta$ and $\left.\mathbf{b}\right)$ are not related to $t$.

The action force $\mathbf{F}$ exerted on a particle by the liquid may be related to the stochastic particle velocity $\mathbf{v}$. As a result, the operators such as $\lambda, \beta$ and $\mathbf{b}$ are also probably related to $\mathbf{v}$. For the sake of simplicity, we assume $\mathbf{F}$ and all the other operators are independent of $\mathbf{v}$, which means a substitution of $\mathbf{v}$ by a statistical mean velocity. In such a case, combining Eq. 9 and Eq. 2 , we can obtain equations as follows

$\frac{\partial \beta}{\partial x_{i}}=0$

$b_{i} F_{i}=0$

$\frac{\partial b_{i}}{\partial x_{j}}+\frac{\partial b_{i}}{\partial x_{i}}=0$ 
$\frac{\partial \lambda}{\partial x_{i}}-m \beta F_{i}=0$

in which $i, j=1,2,3, \beta$ is a constant determined from Eq. 10. From Eq. $13, \lambda$ is expressed as

$\lambda=\int m \beta \mathbf{F} \cdot \mathbf{d x}=m \beta \int F_{i} d x_{i}$

then, a final determination of $\lambda$ is based on a given $F_{i}$. Rewriting Eq. 9, we obtain

$f=\exp \left(\lambda+b_{i} v_{i}-\frac{1}{2} \beta m v^{2}\right)$

$f=A \exp \left(\lambda-\frac{1}{2} m \beta V^{2}\right)$

then, by analogizing the method used in the kinetic theory of gases, we obtain the equation of velocity distribution function of particles for equilibrium state or for dilute steady state in two-phase flow, which has the same form with that of molecules in similar conditions. Here $A=\exp [(1 /$ 2) $\left.m \beta \Sigma\left(v_{o i}\right)^{2}\right], V^{2}=\mathbf{V} \cdot \mathbf{V}=\Sigma\left(v-v_{o i}\right)^{2}, v_{o i}=b_{i} / \beta m$, and $\mathbf{V}$ is the peculiar velocity of a particle. Five parameters of $A, \lambda, \beta, v_{01}, v_{02}, v_{03}$ are left to be determined, and $\lambda$ can be found from Eq. 14 .

Perhaps the major difference in connotation between the velocity distribution function of molecules in the kinetic theory of gases and that of particles in particle-liquid flows is the consideration of the actions of collisions and external forces. Generally speaking, action of collisions among molecules is the most eminent factor in the former and the external forces can be neglected, but the circumstance for the latter is just opposite in dilute steady two-phase flow because of very few collisions and the eminent action of external forces. For flows with high concentration, both the action of collisions and that of external forces must be fully considered.

If the velocity distribution function $f$ can be determined, all the parameters on the movement of particles will be given correspondingly

$n=\int f d v_{i}$

$\vec{v}_{p}=\frac{1}{n} \int \vec{v} f d v_{i}$

$\overline{V_{p}^{2}}=\frac{1}{n} \int\left(\vec{v}-\vec{v}_{p}\right)^{2} f d v_{i}$

Here $n=$ number of particles per unit volume; $\vec{v}_{p}$ and $\overline{V_{p}^{2}}=$ mean velocity and mean square value of the fluctuating velocity of particles, respectively.

It is very difficult for us to determine all the parameters in Eq. 15, but if the concentration distribution of a particle is discussed alone, the problem will be greatly simplified because only the unknown parameter $A$ needs to be given. This can be done by giving a reference concentration $n_{a}$ near the boundary or the vertical mean concentration $\bar{n}$. 


\section{Vertical Concentration Distribution}

From the previous analysis, if we assume that the parameters are not related to the stochastic velocity $\vec{v}$ of the particles, then the following equations can be obtained

$$
\begin{aligned}
& n=\int_{-\infty}^{+\infty} f d v_{i}=A\left(\frac{2 \pi}{m \beta}\right)^{3 / 2} e^{\lambda} \ldots \ldots \\
& \frac{1}{2} m \overline{V_{p}^{2}}=\frac{m}{2 n} \int_{-\infty}^{+\infty}\left(\vec{v}-\vec{v}_{p}\right) f d v_{i}=\frac{3}{2 \beta}
\end{aligned}
$$

For a two-dimensional flow, $\lambda$ is given by

$\lambda=m \beta \int F_{i} d x_{i}=m \beta \int\left(F_{x} d x+F_{y} d y\right)$

$\lambda=m \beta\left(\int F_{y} d y+c\right)=m \beta \int F_{y} d y$

with the constant $c$ being zero. In addition, note that $\overline{V_{p}^{2}}$ in equation

$m \beta=\frac{3}{\overline{V_{p}^{2}}}$.

is unknown, a relation of

$\frac{3}{\bar{V}_{p}^{2}}=\frac{\alpha}{U_{*}^{2}}$

is assumed and Eq. 22 becomes

$m \beta=\frac{\alpha}{U_{*}^{2}}$

where $U_{*}$ is shear velocity, and $\alpha$ is a coefficient.

Since $F_{y}$, the total vertical action force per unit mass on a particle, consists of many forces, such as drag force, lift force, gravitational force, added mass force, and pressure gradient force, etc., we can't expect to determine each force individually. In fact, even though the effects of interaction forces due to acceleration, rotation, etc., have been discussed in the literature, they have not been included in any successful treatment of theoretical analysis of the flow problems of particle-liquid system. Usually, the added mass force can be neglected for the case of small acceleration of particles, and also the action from the collisions between particles can be ignored for dilute particle concentrations. Based on these considerations, all the vertical forces acting on a particle by the fluid can be taken as a comprehensive force, known as comprehensive lift force. According to Owen (1969), the comprehensive lift force by the fluid on a particle can be expressed as

$L_{f} \sim \rho_{f}\left(U_{f}-U_{p}\right) D^{3} \frac{d U_{f}}{d y} \sim \frac{\rho_{f}}{\rho_{p}} m \frac{d U_{f}}{d y}\left(U_{f}-U_{p}\right)=\alpha^{\prime}\left(\frac{\rho_{f}}{\rho_{p}}\right) m \frac{U_{*}^{2}}{y} \ldots \ldots \ldots$

then we obtain

$m F_{y}=\alpha^{\prime}\left(\frac{\rho_{f}}{\rho_{p}}\right) m \frac{U_{*}^{2}}{y}-\frac{\pi}{6} D^{3}\left(\rho_{p}-\rho_{f}\right) g$ 
$F_{y}=\alpha^{\prime}\left(\frac{\rho_{f}}{\rho_{p}}\right) \frac{U_{*}^{2}}{y}-\left(1-\frac{\rho_{f}}{\rho_{p}}\right) \mathbf{g}$

in which, the first term in the right-hand side is the comprehensive lift force and the second is the effective gravitational force of the particle; $\rho_{f}$ and $\rho_{p}$ $=$ density of fluid and particle, respectively; $D=$ the particle diameter, $U_{f}$ and $U_{p}$ are the $x$-direction velocity of fluid and particle.

Based on this analysis, we have

$$
\begin{aligned}
& \lambda=\frac{\alpha}{U_{*}^{2}} \int F_{y} d y=\alpha^{\prime} \alpha \frac{\rho_{f}}{\rho_{p}} \ln y-\alpha\left(1-\frac{\rho_{f}}{\rho_{p}}\right) \frac{g y}{U_{*}^{2}} \\
& =-Z_{*} \eta(\zeta-1) \ln (H \eta) \ldots \ldots \ldots \ldots \ldots
\end{aligned}
$$

Here $\eta=y / H$ and $H$ is a characteristic length, representing flow depth in open channel and pipe radius in pipelines. $Z_{*}$ and $\zeta$ are defined as

$Z_{*}=\alpha\left(1-\frac{\rho_{f}}{\rho_{p}}\right) \frac{\mathrm{g} H}{U_{*}^{2}}$.

$\zeta=\alpha^{\prime} \alpha \frac{\rho_{f}}{\rho_{p}}+1$

From Eqs. 19 and 28, a formula for the vertical concentration distribution of particle numbers per unit volume is given by

$n=A\left(\frac{2 \pi}{\beta m}\right)^{3 / 2}(H \eta)^{\zeta-1} e^{-z_{*} \eta}$

when a reference concentration $n_{a}$ at $\eta_{a}$ is given, the coefficient $A$ in Eq. 31 is thus determined, then the following relation is obtained

$\frac{n}{n_{a}}=\left(\frac{\eta}{\eta_{a}}\right)^{\zeta-1} e^{-z_{*}\left(\eta-\eta_{a}\right)}$

and the vertical mean concentration of particles is

$\bar{n}=\frac{1}{H} \int_{0}^{H} n d y=\int_{0}^{1} n d \eta$

$\bar{n}=n_{a} \eta_{a}^{1-\zeta} e^{Z_{*} \eta_{a}} \gamma\left(\zeta, Z_{*}\right)$

in which

$\gamma\left(\zeta, Z_{*}\right)=\int_{0}^{1} \eta^{\zeta-1} e^{-z_{*} \eta} d \eta$

finally, we obtain a theoretical model for the vertical concentration distribution of particles

$\frac{n}{\bar{n}}=\frac{Z_{*}}{\gamma\left(\zeta, Z_{*}\right)} \eta^{\zeta-1} e^{-Z_{*} \eta}$

Letting $d n / d \eta=0$, the location of maximum concentration along the vertical is determined by 
$\eta_{m}=\frac{(\zeta-1)}{Z_{*}}$

and when $Z_{*}=6\left(\omega_{o} / K U_{*}\right)$ (here $K$ is Karman coefficient and $\omega_{o}$ is settling velocity in still water) and $\zeta=1$ is taken, a typical result for the pattern II distribution in sediment research can be derived

$\frac{n}{n_{a}}=e^{-6\left(\omega_{o} / K U_{*}\right)\left(\eta-\eta_{a}\right)}$

which is the so-called Lane-Kalinske formula tested by the measured data in river hydraulics. It is clear that Eq. 37 is only a special case of Eq. 32.

\section{Comparison WITH Data}

To test the relation (Eq. 35), we made a series of experiments on the particle-liquid flows in a $4 \times 6 \mathrm{~cm}^{2}$ square pipe by means of LDA. The fluid and particle properties with some experimental results are given in $\mathrm{Ta}$ ble 1 , in which, $U_{*}=$ shear velocity; $C=(\pi / 6) D^{3} \bar{n}$, the vertical mean volumetric concentration. All the particles used in the experiments are spherical plastic. The results of comparison are shown in Fig. 2 with given parameters $Z_{*}$ and $\zeta$

$$
\begin{gathered}
Z_{*}=5 \frac{\omega_{o}}{U_{*}} \ldots \ldots \\
\zeta=6 \frac{D}{H \sqrt{\frac{\rho_{p}}{\rho_{f}}}}+1
\end{gathered}
$$
35 ).

It is clear that all the measured data agree very well with the relation (Eq.

It should be stressed that there indeed are some differences between the particle concentration distribution in pipeline flow and that in open channel flow. Generally speaking, the former is more uniformly distributed than the latter under the similar flow conditions. When the data measured in open

\begin{tabular}{|c|c|c|c|c|c|c|c|c|}
\hline \multirow[b]{2}{*}{$\begin{array}{c}\text { Run } \\
\text { number } \\
\text { (1) }\end{array}$} & \multirow{2}{*}{$\begin{array}{c}\text { Mean } \\
\text { diameter } \\
D(\mathrm{~mm}) \\
(2) \\
\end{array}$} & \multirow[b]{2}{*}{$\begin{array}{c}\text { Diameter } \\
D(\mathrm{~mm}) \\
(3)\end{array}$} & \multirow[b]{2}{*}{$\begin{array}{c}\text { Density } \rho_{p} \\
\left(\mathrm{gr} / \mathrm{cm}^{3}\right) \\
(4)\end{array}$} & \multirow{2}{*}{$\begin{array}{l}\text { Settling } \\
\text { velocity } \omega_{o} \\
(\mathrm{~cm} / \mathrm{s}) \\
(5)\end{array}$} & \multicolumn{2}{|c|}{ Series A } & \multicolumn{2}{|c|}{ Series B } \\
\hline & & & & & $\begin{array}{c}C \\
\cdot(\%) \\
(6)\end{array}$ & $\begin{array}{c}U_{*} \\
(\mathrm{~cm} / \mathrm{s}) \\
(7)\end{array}$ & $\begin{array}{c}C \\
(\%) \\
(8)\end{array}$ & $\begin{array}{c}U_{*} \\
(\mathrm{~cm} / \mathrm{s}) \\
(9)\end{array}$ \\
\hline 1 & 1.8 & $1.6-2.0$ & 1.05 & 2.56 & 0.330 & 3.28 & 0.130 & 6.13 \\
\hline 2 & 1.3 & 1.0 & 1.05 & 2.17 & 0.440 & 3.35 & 0.570 & 6.00 \\
\hline 3 & 1.4 & $1.2-1.6$ & 1.60 & 6.90 & 0.310 & 4.76 & 0.198 & 6.11 \\
\hline 4 & 1.1 & $1.0-1.2$ & 1.60 & 5.15 & 0.240 & 4.52 & 0.200 & 6.15 \\
\hline 5 & 0.85 & $0.7-1.0$ & 1.60 & 4.51 & 0.097 & 4.79 & 0.194 & 6.33 \\
\hline 6 & 0.6 & $0.5-0.7$ & 1.60 & 3.79 & 0.057 & 4.90 & 0.042 & 6.23 \\
\hline 7 & 2.29 & 2.29 & 1.34 & 6.15 & 0.440 & 4.83 & 0.510 & 6.17 \\
\hline
\end{tabular}

TABLE 1. Descriptions of Experimental Data 

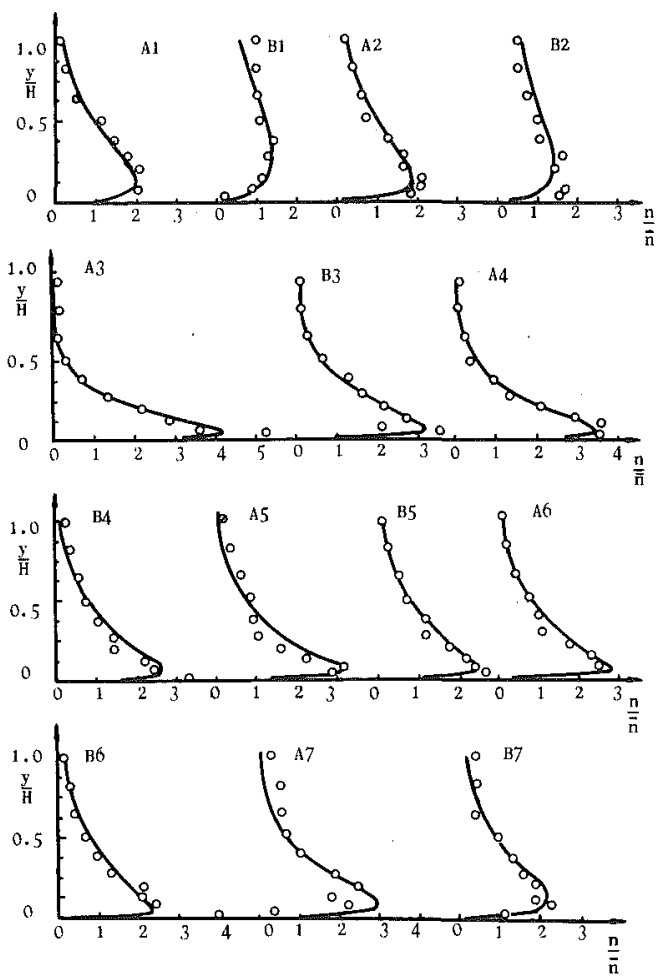

FIG. 2. Comparisons of Eq. 35 and PIpeline Data

channel flow by Bouvard and Petkovic (1985) are compared with Eq. 35, this argument is confirmed. In such a case, expressions for $Z_{*}$ and $\zeta$ must be recast

$$
\begin{gathered}
Z_{*}=15 \frac{\omega_{o}}{U_{*}} \ldots \\
\zeta=18 \frac{D}{H \sqrt{\frac{\rho_{p}}{\rho_{f}}}}+1
\end{gathered}
$$

With these two parameters, we obtained very good agreement between Eq. 35 and Bouvard data, as shown in Fig. 3.

From these mentioned discussions, the patterns for particle concentration distribution is determined by the parameters $Z_{*}$ and $\zeta$. When $\zeta=1$ is taken, the ordinary pattern II distribution is really true, but for larger $\zeta$, the pattern I distribution more frequently occurs. Until now, the cause of formation for the two patterns of concentration distribution has been explained by attributing it to the lift force from the simple-kinetic-theory point of view, in such a way that all the fluid and particle properties have been considered reason- 


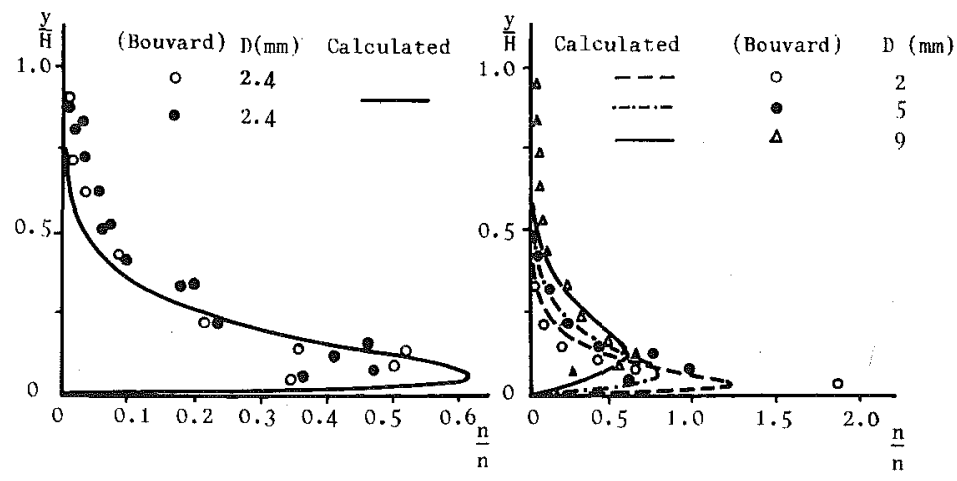

FIG. 3. Comparisons of Eq. 35 and Channel Data

ably. This makes a possibility of evaluating the particle concentration distribution quantitatively.

\section{Conclusion}

Based on kinetic theory in two-phase flow, the law of vertical distribution for dilute particle concentration has been discussed from the angle of microscopic descriptions of mechanism. The main contributions made in this paper are as follows:

1. The vertical distributions of particle concentration are classified into two kinds of patterns (i.e., pattern I and pattern II), and the mechanism for the existence of two kinds of concentration distributions is given in a reasonable way. The cause of formation for the pattern I and pattern II is mainly attributed to the lift force on the particles by the fluid.

2. By analogizing the method adopted in the kinetic theory of gases, the velocity distribution function of particles is obtained. As a result, a theoretical model for describing the pattern I and pattern II distributions is presented. All the data measured by means of LDA agree very well with the present model. This is a rare achievement for earlier models.

3. The pattern I distributions frequently appear for large light particles and the positions for maximum concentration in a vertical line rise with the increase of shear velocity. For small heavy particles, the pattern II distributions are commonly formed.

4. The observations made both in open channels and pipelines show that particle concentration distributions in pipelines are more uniform than those in open channels.

\section{Appendix I. References}

Bouvard, M., and Petkovic, S. (1985). "Vertical dispersion of spherical, heavy particles in turbulent open channel flow." J. Hydr. Res., 23, 5-19.

Chapman, S., and Cowling, T. G. (1970). The mathematical theory of non-uniform gases. Third Ed. Cambridge University Press, Cambridge, England.

Dai, J. L. (1985). "Two-phase pipe flow with a bottom layer of bed-load," thesis 
presented to Tsinghua University, at Beijing, China, in partial fulfillment of the requirements for the degree of Doctor of Philosophy.

Graf, W. H. (1971). Hydraulics of sediment transport, McGraw-Hill Book Company, New York, N.Y., 172.

Michalik, M. A. (1973). "Density patterns of the inhomogeneous liquid in the industrial pipe-lines measured by means of radiometric scanning." La Houille Blanche, $1,53-57$.

Ni, J. R., and Hui, Y. J. (1988). "Theories of vertical concentration distribution of suspended sediment and their relationships." J. Nanjing Hydr. Inst., 1, 83-97 (in Chinese).

Ni, J. R. (1989). "A generalized formula on the vertical distribution of suspended sediment concentration." Proc. of the Fourth Int. Symp. on River Sedimentation, Beijing, China, 1, 603-610.

Ni, J. R., and Wang, G. Q. (1987). "On the two patterns of vertical distribution of sediment concentration and their formation cause." J. Hydr. Engrg., 7, 60-68 (in Chinese).

Owen, P. R. (1969). "Pneumatic transport." J. Fluid Mech., 39, 407-432.

Wang, G. Q. (1989). "The kinetic theory with experimental studies for the solid/ liquid two-phase flow and granular flow," thesis presented to the Tsinghua University, at Beijing, China, in partial fulfillment of the requirements for the degree of Doctor of Philosophy.

\section{Appendix II. Notation}

The following symbols are used in this paper:

$D=$ particle diameter;

$f=$ velocity distribution function of particles;

$F_{i}=$ the unit mass force on a particle;

$F_{y}=$ the vertical component of $F_{i}$;

$\mathrm{g}=$ gravitational acceleration;

$H=$ characteristic length;

$K=$ Karman coefficient;

$L_{f}=$ comprehensive lift force on a particle;

$m=$ mass of a particle;

$n=$ particle numbers per unit volume;

$\bar{n}=$ vertical mean particle numbers per unit volume;

$n_{a}=$ particle numbers per unit volume at the position $a$;

$t=$ time;

$U_{f}=$ fluid velocity component in $x$-direction;

$U_{P}=$ particle velocity component in $x$-direction;

$U_{*}=$ shear velocity;

$\vec{V}=$ peculiar velocity of a particle;

$\vec{v}_{p}=$ mean velocity of particles;

$v_{i}=$ the particle stochastic velocity;

$v_{o i}=$ characteristic velocity;

$x_{i}=$ spatial position;

$Z_{*}=$ parameter related to suspension index;

$\alpha=$ coefficient;

$\alpha^{\prime}=$ coefficient related to lift force on a particle;

$\beta=$ Lagrange operator;

$\lambda^{\prime}=$ Lagrange operator; 

$\lambda=\lambda^{\prime}-1$
$\eta=y / H$
$\rho_{f}=$ density of fluid;
$\rho_{p}=$ density of particle;
$\omega_{o}=$ settling velocity; and
$\zeta=$ parameter defined in the text. 